Notre Dame Journal of Formal Logic

Volume 21, Number 4, October 1980

\title{
Boole's Criteria for Validity and Invalidity
}

\author{
JOHN CORCORAN and SUSAN WOOD
}

The mathematics we have to construct are the mathematics of the human intellect.-Boole, 1847

It is one thing for a given proposition to follow or to not follow from a given set of propositions and it is quite another thing for it to be shown either that the given proposition follows or that it does not follow.* Using a formal deduction to show that a conclusion follows and using a countermodel to show that a conclusion does not follow are both traditional practices recognized by Aristotle and used down through the history of logic. These practices presuppose, respectively, a criterion of validity and a criterion of invalidity each of which has been extended and refined by modern logicians: deductions are studied in formal syntax (proof theory) and countermodels are studied in formal semantics (model theory).

The purpose of this paper is to compare these two criteria to the corresponding criteria employed in Boole's first logical work, The Mathematical

\footnotetext{
*The authors thank James van Evra (University of Waterloo) for help over a period of years. Some of the ideas in this paper are from the second author's doctoral dissertation [26] which was supervised by the first author. Van Evra was the outside reader. A National Science Foundation grant enabled the second author to read the unpublished Boole manuscripts on deposit in the Library of the Royal Society of London. Wendy Ebersberger, John Kearns, William Parry, Charles Lambros, and, especially, Michael Scanlan (all of SUNY/Buffalo) gave suggestions, criticisms, and friendly support and cooperation. Earlier versions were presented to the Buffalo Logic Colloquium (November 1977), to the Association for Symbolic Logic (April 1978, see [12]), and to the Philadelphia Logic Colloquium (April 1979). Finally, the authors are grateful for conscientious and penetrating criticism to the editor and to anonymous referees.
} 
Analysis of Logic (1847). In particular, this paper presents a detailed study of the relevant metalogical passages and an analysis of Boole's symbolic derivations.

It is well-known, of course, that Boole's logical analysis of compound terms (involving 'not', 'and', 'or', 'except', etc.) contributed to the enlargement of the class of propositions and arguments formally treatable in logic. The present study shows, in addition, that Boole made significant contributions to the study of deductive reasoning. He identified the role of logical axioms (as opposed to inference rules) in formal deductions, he conceived of the idea of an axiomatic deductive system (which yields logical truths by itself and which yields consequences when applied to arbitrary premises). Nevertheless, surprisingly, Boole's attempt to implement his idea of an axiomatic deductive system involved striking omissions and Boole does not use his own formal deductions to establish validity. Boole does give symbolic derivations, several of which are vitiated by the fallacy of supposing that a solution to an equation is necessarily a logical consequence of the equation. This fallacy seems to have led Boole to confuse equational calculi (i.e., methods for generating solutions) with proof procedures (i.e., methods for generating consequences). The methodological confusion is closely related to the fact, shown in detail below, that Boole had adopted an unsound criterion of validity.

It is also shown that Boole totally ignored the countermodel criterion of invalidity. Careful examination of the text does not reveal with certainty a test for invalidity which was adopted by Boole. However, we have isolated a test that he seems to use in this way and we show that this test is ineffectual in the sense that it does not serve to identify invalid arguments.

This paper goes beyond the simple goal stated above. Besides comparing Boole's earliest criteria of validity and invalidity with those traditionally (and still generally) employed, this paper also investigates the framework and details of The Mathematical Analysis of Logic (MAL). The investigation is carried out using the context and methods of modern logic. The main object of the investigation is the discovery and exact articulation in modern terms of the objective logical and mathematical content of Boole's first logical work. It is worth emphasizing that no attempt has been made here to characterize a distinctly Boolean doctrine that pervades Boole's logical works as a whole nor has any attempt been made to establish Boole's proper role in the historical development of logic. It is true, however, that some references have been made not only to Boole's later writings on logic and mathematics but also to the views of his predecessors, his contemporaries and his successors. With few exceptions, references to material outside of $M A L$ are made solely for purposes of comparison. In a few places, all clearly noted, ambiguity and vagueness of passages in $M A L$ are tentatively treated by comparison with his later works.

Boole wrote several things on logic after $M A L$ and the later writings contain significant changes (see e.g., [15], p. 205). It is not clear how long Boole spent developing the ideas reported in $M A L$ but it is clear that Boole did not spend more than a few months at the most on writing it (see $M A L$ [3], Preface). In the preface to $M A L$ Boole says that he had thought about the subject previously "at different periods" but in the preface to Laws of Thought (1859) he says that $M A L$ "was written within a few weeks after its idea had 
been conceived". In an 1851 article ([6], p. 252) Boole refers to $M A L$ as "a hasty and (for this reason) regretted publication". Thus, there is reason to believe that $M A L$ does not represent Boole's mature, complete, or final views, and criticisms of $M A L$ cannot be taken as criticisms of Boole's work as a whole.

Nevertheless there are good reasons for seeking understanding of the objective logical and mathematical content of $M A L$. In the first place, an understanding of the development of Boole's thought throughout his logical career requires understanding of his thought at each stage. In the second place, without a clear statement of the facts involved one cannot assume that every change in Boole's thinking was objectively an improvement. In the third place, even if one's interest is limited to Boole's mature and final logical views there is reason to believe that an understanding of what these are and what these are not will be enhanced by comparison with his earliest thinking. The latter is particularly likely because, although later works do incorporate changes, Boole never identifies what he takes to be errors in $M A L$ and, in most cases, he does not even identify the changes.

One device repeatedly used in this article to illustrate and characterize Boole's logic is comparison of it with Aristotle's logic. Such a comparison may appear to modern logicians as far-fetched, irrelevant, and perhaps even reactionary. This initial negative response is largely justified from a strictly modern and nonhistorical point of view. Moreover it is also partly justified from the point of view of historians whose interest is to see what Boole contributed to modern logic. However if one's interest is to understand Boole's place in the development of logic and to prepare the evidence necessary to assess Boole as a logician then it is Aristotelian logic which is par excellence the system to which Boole's logic is to be compared. There are several reasons for this.

In the first place, Aristotelian logic so dominated logical thinking up to Boole's time that the subject of logic was widely considered to be virtually identical to the Aristotelian doctrine. It was precisely within Boole's time, and partly a result of Boole's work, that Aristotle's influence began to be replaced with a more modern outlook. In the second place, Boole (not to mention DeMorgan, Venn, and even Whitehead) devoted much effort to reformulating Aristotelian logic. Roughly one-third of $M A L$ concerns categorical propositions and syllogisms. Besides, Boole himself $(M A L$, p. 13) literally invites the comparison. In the third place, it is now clear ([2], [10], [21]) that Aristotle's basic outlook is very close to the modern viewpoint especially as formulated by Church [7] and Tarski [22], and, thus, without serious distortion, it might be said that a contemporary examination of Boole's logic cannot avoid comparison, at least indirectly, with Aristotle's logic.

1 Preliminaries A set of propositions $P$ is said to logically imply a single proposition $c$ if it is logically impossible that all propositions in $P$ be true while $c$ be false. Saying that $c$ follows from $P$, or is a consequence of $P$, is the same as saying that $P$ implies $c$. To connect these ideas to the traditional terminology of arguments, premises, and conclusions, define a premise-conclusion argument to be a set, $P$, of propositions called the premises together with a single proposition called the conclusion. For purposes of abbreviation let $(P, c)$ be the argument with premises $P$ and conclusion $c$. To say that $(P, c)$ is valid is to say that 
$P$ logically implies $c$, and, of course, to say that $(P, c)$ is invalid is to say that $P$ does not logically imply $c$.

If one considers only trivial arguments such as the two-premise syllogism then the problem of producing plausible criteria for validity and invalidity does not seem demanding or even interesting. But the situation is otherwise if one considers more complex arguments, such as those found in philosophical or mathematical discourse. For example, if one takes as premise sets various subsets of the axioms of Euclidean geometry and as conclusions various geometrical theorems and the negations of various axioms, then in raising the problem of criteria for validity and invalidity one is implicitly raising a host of traditional questions, including the question of criteria for the independence of the axioms of geometry.

At any rate, Aristotle noticed that the validity of an argument $(P, c)$ can be established by means of a step-by-step deduction interpolating between $P$ and $c$ intermediate propositions which clearly link the conclusion to the premises. Thus by presenting a set of rules of inference for inserting intermediate propositions on the basis of those already set down and by indicating how these rules are used to construct deductions, Aristotle presented a criterion for validity.

Let us call Aristotle's rules A-rules. Consider a list of propositions beginning with all or some of the members of $P$, whose subsequent lines are obtained by applying $A$-rules to previous lines, and which ends with $c$. Such a list is called a direct $A$-deduction of $c$ from $P$. An indirect $A$-deduction of $c$ from $P$ is a direct $A$-deduction which begins with members of $P$ and the contradictory of $c$ and which ends in a contradiction. Aristotle took the existence of an $A$-deduction of $c$ from $P$, direct or indirect, as a criterion for validity. ${ }^{1}$ Using modern terminology, Aristotle's criterion of validity can be restated as follows: if $(P, c)$ is $A$-deducible then $(P, c)$ is valid.

It is, of course, possible to agree with Aristotle that deducibility is a criterion of validity while disagreeing with his specific definition of "deduction". For example, one might wish either to delete rules or to add rules or both. In particular, the logicians who believe that "Some animals are dogs" does not follow from "All dogs are animals" would want to delete one of Aristotle's rules. Likewise, since "All nonanimals are nondogs" is not deducible in Aristotle's system from "All dogs are animals", one might want to add a rule. Indeed, this is one of the changes suggested by Boole $(M A L$, pp. 26, 28, 34).

Aristotle also noticed that the invalidity of an argument $(P, c)$ can be established by exhibiting another argument $\left(P^{*}, c^{*}\right)$ which $(1)$ has the same form as $(P, c)$ but which $(2)$ has premises known to be true and a conclusion known to be false. For example, the second argument below (A2) establishes the invalidity of the argument immediately below (A1).

A1 All maples are plants.

All maples are trees.

?All trees are plants.

A2 All maples are trees.

All maples are plants.

?All plants are trees. 
Aristotle took two arguments to have the same form if one can be converted into the other by replacing in a one-one fashion the "content words" of one with those of the other. The replacement (maples $\rightarrow$ maples, plants $\rightarrow$ trees, trees $\rightarrow$ plants) converts A1 into A2.

This method has been used down through the history of logic. For example, it was used in the medieval period by Anselm (with particular dexterity in De Grammatico [1]). In the 19th century it was used by Beltrami to establish that the parallel postulate is not implied by the other axioms of geometry (Church [7], p. 328), and in this century it was used by Cohen to establish the fact that the continuum hypothesis is not a consequence of the axioms of set theory [8].

A counterargument to an argument $(P, c)$ is an argument $\left(P^{*}, c^{*}\right)$ where (1) $\left(P^{*}, c^{*}\right)$ has the same form as $(P, c)$ and $(2)$ the premises $P^{*}$ are all true and the conclusion $c^{*}$ is false. Aristotle took a counterargument to establish the invalidity of a given argument. Aristotle's criterion for invalidity can be restated as follows: if $(P, c)$ has a counterargument then $(P, c)$ is invalid.

The striking asymmetry of the two criteria has been remarked on before (e.g., Tarski [22], pp. 117-125). To establish validity one uses a logical deduction; to establish invalidity one appeals to facts (uses a counterargument or, equivalently, a countermodel ${ }^{2}$ ).

Deduction is usefully construed as a chaining together of evidently valid arguments. The method of refutation by counterargument is virtually implicit in the so-called principle of logical form: an argument is valid or invalid according to its form, i.e., two arguments in the same form are both valid or both invalid.

2 Boole on establishing validity As indicated above, the traditional approach to logic focuses on the problem of classifying a given argument as valid or invalid. The premises and the conclusion are given, not as true, but simply as constituting a premise-conclusion argument, and the role of logic, so to speak, is to determine whether this given argument is valid or invalid, whether the conclusion follows or does not follow from the premises.

In regard to validity Boole presented a deductive system which he took as $a$ criterion of validity. But there are several qualifications to be made.

In the first place, he seemed to take the primary role of logic to be, not the determination of validity and invalidity of given arguments, but rather the determination of (unknown) consequences of given premises. Instead of classifying given arguments (whose conclusions are given) he focuses on methods of "generating" the consequences of given premises. It is as if Boole took deducing to be the producing of consequences rather than the producing of deductions (cf. [6], pp. 218, 232, 238, 239). Boole's paradigm, then, is not to be found in the logical practice of producing deductions, given the premises (or axioms) and given a conjectured conclusion. Rather, Boole looked toward the mathematical practice of finding a solution to a given set of equations ( $M A L$, pp. 32ff).

Boole did not, however, ignore the traditional concern for producing deductions ([6], p. 142). His deductive system (MAL, pp. 15ff) can be used for producing deductions of given conclusions from given premises. In later works he refers to "the synthetical method" ([6], pp. 231, 232) wherein "we begin with the premises ... and . . arrive by a direct process at the conclusion ...". 
But Boole did not emphasize the deductive system as a criterion of validity. Indeed, he sometimes employs a different and incorrect criterion (see below) even when he could have used his own deductive method correctly and straightforwardly. Moreover, the presentation of the deductive system is perfunctory, and the system has deficiencies which Boole himself could have recognized easily.

2.1 The character of Boole's deductive system For Boole "logical propositions", including the categorical propositions studied by Aristotle, are identities (equations) between classes ( $M A L$, pp. 8, 31, 32). He used 0 to denote the null set and 1 for the Universe of all entities. His "elective symbols", $x, y, z, u, v$, and $w$, were used to indicate arbitrary but fixed classes, empty and nonempty alike; but, as will be seen below, he often reserves the symbol $v$ for a special use in connection with nonempty classes.

Boole nowhere explicitly asserts that the elective symbols can indicate empty sets, nor does he ever explicitly say that they cannot indicate empty sets. In passages only indirectly related to present concerns he writes " $x=0$ " $(M A L$, p. 51). However, there are many passages, to be mentioned below, where fallacies exist when elective symbols are able to have empty sets as values. On the other hand, if Boole is to be consistently interpreted as having restricted the values of the elective symbols to nonempty sets then there are many laws involving the empty set which he has not stated or implied (e.g., $0+0=0,0.0=0$ ), and the device involving $v$ which is widely supposed to have been invented to explicitly state nonemptiness seems redundant ([15], p. 205).

It is particularly important in this connection to note that Boole later believed that the formal laws of logic are those principles which hold of the null set and the universal set ([6], pp. 20, 211, 218). Thus even if the elective symbols are not allowed to indicate the null set, his propositions and inferences involving the elective symbols may have been supposed by Boole to hold when 0 and 1 are arbitrarily substituted for them.

In $M A L,+$ seems to resemble union, and - seems to resemble relative complement. Juxtaposition (or concatenation) was taken to indicate intersection. Unions and complements are often (but not always, e.g., $M A L$, pp. 43, $53,58)$ grouped with parentheses.

It is to be emphasized both that Boole gave nothing which could be interpreted as a formal grammar and that determination of his semantics is highly problematic. Not only is the use of the symbol $v$ unclear but there may be no interpretation for + and - which is consistent with $M A L$ as a whole. ${ }^{3}$

The vagueness and incompleteness of his definition of his semantics contrasts sharply with his philosophical comments ${ }^{4}$ that ". . . symbols are used with a full understanding of their meaning, with a perfect comprehension of that which renders their use lawful ...".

Boole recognized logical truths, formulas which, like $x=x$, are true regardless of which classes are denoted by their elective symbols. Although he has no term in $M A L$ for logical truth simpliciter it is virtually certain that he had the idea: he speaks of relations among the operations ". . . whether as respects the repetition of a given operation or the succession of different ones, or some other particular, which are never violated" $(M A L, \mathrm{p}$. 5). In later 
writings ([6], p. 215), Boole refers to "propositions true in consequence of their form alone" and he gives as examples "A man is a man" and "A man is either a tree or not a tree". Among the logical truths he singles out some which are "obvious" (MAL, p. 6). His obvious logical truths are later referred to as laws (MAL, pp. 16-18) and are used as logical axioms.

Accordingly, Boole's deductions are not merely sequences of propositions derived from premises by means of rules but they also contain logical axioms. This is a striking qualitative improvement on Aristotle's direct deductions which were constructed using eight rules (the three conversions, the four "perfect syllogisms", and the rule of repetition) but no logical axioms (Corcoran [10], [11]). Boole was aware that he was breaking new ground by his discovery of certain logical truths $(M A L, \mathrm{p} .6)$ but in $M A L$ he did not seem to realize the novelty of using them in deductions.

Nevertheless in later works (cf. [6], pp. 215, 216) Boole is clear about the fact that deductions are constructed by interpolating logical axioms and by applying rules of inference. He says "the laws of thought include . . formal laws of judgment and formal laws of reasoning". 5 (Also see [4], pp. 101-102.)

Speaking loosely for the moment, one can say that Boole presented a system of deductions based on one rule (a version of equals-substituted-forequals) and four logical axioms (or axiom schemes). Boole was explicitly aware of only some contrasts between his own system and that of Aristotle. He compares his own rule with the so-called Aristotelian dictum de omni (which had been supposed by some to be the basic rule of Aristotle's logic) and he refers to his system as "another mode of considering the subject" ( $M A L$, p. 18). In later works (cf. [6], pp. 25, 237, 238) he argues that rules of inference alone would not be sufficient for reasoning and that "we are compelled to have recourse to ... canons ... which enable us to add to the premises, whose truth is only assumed, other propositions whose truth is not assumed but necessary".

One thing that Boole did not explicitly notice (and probably did not notice at all) is that Aristotle's system included indirect deductions whereas his own did not. It is important to realize that Boole's deductive system necessarily lacked indirect deductions because his language lacked negation. ${ }^{6}$ Likewise, it is important to realize that Aristotle's deductive system necessarily lacked logical axioms because his language lacked logical truths.

As far as the analysis of the forms of deduction is concerned, Boole's system was a step forward in one respect and a step backward in another; logical axioms were added but indirect deductions were dropped.

Before going on to consider the details of Boole's deductive system there is one final point of comparison between Boole and Aristotle which should be mentioned. Aristotle emphasizes the epistemic or knowledge-producing property of deductions: by means of a deduction one comes to know that the conclusion follows. Aristotle says that a deduction "makes clear" the logical connection of the conclusion to the premises. In $M A L$, however, there is no explicit reference to the epistemic nature of deduction.

2.2 The details of Boole's deductive system Boole presents his deductive system in four pages (MAL, pp. 16-19). After stating his logical axioms (or 
"laws", to use his term) he says that they are "sufficient for the basis of a calculus" (MAL, p. 18).

For convenience we restate his logical axioms and give them names.

Axioms:

$\begin{array}{lll}\text { I } & x=x & \text { identity } \\ \text { LDM } & x(u+v)=x u+x v & \text { left distribution of multiplication } \\ \text { CM } & x \cdot y=y \cdot x & \text { commutativity of multiplication } \\ \text { IM } & x \cdot x=x & \text { index law }\end{array}$

It is to be emphasized that these are all of the axioms which Boole explicitly lists. Perhaps it was his casual attitude concerning parentheses which led him to overlook the two associative laws.

AM $\quad x \cdot(y \cdot z)=(x \cdot y) \cdot z \quad$ associativity of multiplication

AA $\quad x+(y+z)=(x+y)+z$ associativity of addition

Rule: Boole introduces his rule, which he calls "an axiom", with the following statement: "The one and sufficient axiom ... is that equivalent operations performed upon equivalent subjects produce equivalent results". It is clear that this is to be understood as a single rule encompassing the following three subrules (a point which Boole himself makes in later works ([6], pp. 235, 236 and cf. [4], p. 36).

$\begin{array}{lll}\text { FA } & x=y, z=w / x+z=y+w & \text { functionality of addition } \\ \text { FS } & x=y, z=w / x-z=y-w & \text { functionality of subtraction } \\ \text { FM } & x=y, z=w / x \cdot z=y \cdot w & \text { functionality of multiplication }\end{array}$

This is the only rule of inference that Boole explicitly states as a rule of his system in MAL. Moreover, as mentioned above, he refers to this rule as "the one and sufficient axiom". It would seem then that Boole is overlooking symmetry and transitivity of identity. However in a footnote $(M A L, \mathrm{p} .18)$ he refers to two other rules ${ }^{7}$ in a way which suggests that he thinks that they are already somehow included in the above. One of the latter two rules is irrelevant to the system because it presupposes that the language contains negation. This rule is, of course, never used and it is never referred to again.

The other rule mentioned in the footnote is: "If two terms agree with one and the same third, they agree with each other". We take this to be represented by the following:

$\begin{array}{lll}\mathbf{R} 1 & x=z, y=z / x=y & \text { equal to a third } \\ \mathbf{R} 2 & x=z, z=y / x=y & \text { transitivity }\end{array}$

Boole's statement ${ }^{8}$ of this rule presupposes symmetry of identity, i.e., Boole would not have used the above wording unless he thought that identity is symmetrical. Moreover, one can derive symmetry using $\mathrm{R} 1$ and $\mathrm{I}(x=x)$.

S $\quad x=y / y=x \quad$ symmetry

Although the present authors do not wish to draw the following conclusion, it must be noted that from the language used in $M A L$, together with 
passages in later works (e.g., [6], pp. 231, 235, 236], some logicians may wish to infer that the rule which Boole intended for $M A L$ was not any of the above but rather was the more general principle of substitution of identicals, viz., from $x=y$ and a sentence $S$ infer any sentence obtained from $S$ by erasing all occurrences of $x$ and $y$ and then filling the blanks $a d$ lib with occurrences of $x$ and $y$. Boole seemed to view such identity inferences in a formally different, but equivalent, way ([6], p. 127).

There is little doubt that Boole believed that his system embodied a complete analysis of reasoning involving his fundamental operations. He uses the term "sufficient" twice and in the footnote mentioned above he states:

Any account of the process of reasoning is insufficient which does not represent ... the laws [= logical axioms] of the operation which the mind performs in that process .... It is presumed that the laws in question are adequately represented by the fundamental equations of the present Calculus. The proof of this will be found in its capability of expressing propositions, and of exhibiting in the results of its processes, every result that may be arrived at by ordinary reasoning.

2.3 Omissions from Boole's deductive system The inadequacies of Boole's deductive system are obvious to a casual observer.

Firstly, there are no laws specifically governing the null set and the universal set. Thus one might want to add the following9:

$\begin{array}{ll}\text { N1 } & 0 \cdot x=0 \\ \text { N2 } & 0+x=x \\ \text { U1 } & 1 \cdot x=x \\ \text { U2 } & 1+x=1\end{array}$

Especially notable is the absence of a law that Boole calls "the principle of contradiction" in Laws of Thought, viz.:

NU $\quad x \cdot(1-x)=0 \quad$ principle of contradiction

Secondly, the only axiom governing a reiterated argument and explicitly mentioned by Boole is $x \cdot x=x$. Thus the following are missing.

IA $\quad x+x=x \quad$ index for addition

IS $\quad x-x=0 \quad$ index for subtraction

Thirdly, Boole gives no axioms involving addition alone. We have already mentioned that the index law and the associative law are missing. (The commutative laws are stated only verbally ( $M A L$, pp. 5-6).)

Fourthly, the only distribution law mentioned is that given above. Thus, e.g., the following are missing:
LDA $\quad x+(y \cdot z)=(x+y) \cdot(x+z)$
left distribution of addition
LDMS $\quad x \cdot(y-z)=(x \cdot y)-(x \cdot z)$
left distribution of multiplication over subtraction

Finally, we mention that Boole omits the laws that have come to be named after De Morgan ${ }^{10}$ and the so-called absorption laws. 
For later reference it should be observed that Boole does not reject any of these omitted laws. Indeed, in $M A L$ there are no formulas that he explicitly refers to as not being laws. (But, cf. [4], p. 50.)

The absence of the associative laws can be somewhat explained by Boole's inattention to parentheses (e.g., $M A L$, pp. 43, 53, 58), but the other omissions, taken together, seem to indicate that Boole did not systematically investigate deduction. Boole's remarks concerning the "sufficiency" of his system must be understood as revealing casual beliefs not based either on serious reflection or on organized experience. As we will see below, Boole did not use his own system and he did not expend any energy in studying it prior to publication. Indeed, in the whole of $M A L$ there is not one example of a deduction in the above system.

In the course of his symbolic derivations there are places where he makes inferences which could be derived in a few steps by means of his stated rules. For example the following inferences are all made:

$$
\begin{array}{ll}
x=y / x \cdot z=y \cdot z & \text { multiplying an equation by } z \\
x=y / z \cdot x=z \cdot y & \text { multiplying } z \text { by an equation } \\
x=y / x-z=y-z & \text { subtracting } z \text { from an equation } \\
x=y / z-x=z-y & \text { subtracting an equation from } z
\end{array}
$$

In regard to the production of formally correct deductions and in regard to extensive organized experimentation, Boole's work contrasts sharply with Aristotle's. Aristotle gives many examples of deductions meticulously constructed according to his rules. In fact, in the course of hundreds of applications of rules there are at most a handful of missing lines. In addition, Aristotle experimented with his system in a highly organized fashion. In contrast to Boole, Aristotle's belief in the completeness of his system was not only true but it was based on experimentation and serious reflection-even though it was not completely justified according to modern standards (Corcoran [10], [11]). On the other hand, as we have seen, Boole's belief in completeness was false and its falsity would have been obvious to Boole if he had seriously considered the question. Naturally, Boole would have added more axioms and rules, and if he had still failed to have a complete system he would have had a system which at least would not be so obviously incomplete.

As Boole indicates $(M A L$, p. 7), his logical investigations were intended to provide a reformulation of "the received logic". Indeed, over one-third of $M A L$ (measured in pages) is devoted to the reformulation of traditional conversions and syllogisms. Moreover, even though Boole's formal language can formulate arguments involving compound terms such as "Europeans or Asiatics" ( $M A L$, p. 58), Boole does not give a single example of such an argument. This seems to show that Boole was not fully aware of the fact that he had discovered a framework essentially richer than Aristotle's. ${ }^{11}$ In this connection it is worth noting that fifty years later when Whitehead [25] came to apply algebra to logic he derived only the traditional two-premise syllogisms omitting derivations of arguments involving compound terms.

At any rate, given that Boole had a new system of deduction and given that he intended to reformulate traditional logic, one would expect to find detailed deductions of some Aristotelian syllogisms. Below are two examples of 
deductions of syllogisms using axioms and rules used by Boole (but not necessarily stated by him).

Example 1

$\begin{array}{rlr}\text { All } Y \mathrm{~s} \text { are } X \mathrm{~s} & \text { 1. } y=y x & \\ \text { All } Z \mathrm{~s} \text { are } Y \mathrm{~s} & \text { 2. } z=z y & \\ \text { ?All } Z \mathrm{~s} \text { are } X \mathrm{~s} & \text { ? } z=z x & \mathrm{I} \\ & \text { 3. } z=z & \mathrm{FM}, 1,3 \\ & \text { 4. } z y=z(y x) & \mathrm{AM} \\ & \text { 5. } z(y x)=(z y) x & \mathrm{R} 2,4,5 \\ & \text { 6. } z y=(z y) x & \mathrm{I} \\ \text { 7. } x=x & \mathrm{FM}, 2,7 \\ \text { 8. } z x=(z y) x & \mathrm{R} 1,6,8 \\ \text { 9. } z y=z x & \mathrm{R} 2,2,9 \\ \text { 10. } z=z x & \end{array}$

Example 2

$\begin{array}{rlr}\text { All } X \mathrm{~s} \text { are } Y \mathrm{~S} & \text { 1. } x=x y & \\ \text { No } Z \mathrm{~s} \text { are } Y \mathrm{~s} & \text { 2. } z y=0 & \\ \text { ?No } Z \mathrm{~s} \text { are } X \mathrm{~s} & \text { ? } z x=0 & \mathrm{I} \\ & \text { 3. } z=z & \mathrm{FM}, 3,1 \\ & \text { 4. } z x=z(x y) & \mathrm{CM} \\ \text { 5. } x y=y x & \mathrm{R} 2,1,5 \\ \text { 6. } x=y x & \mathrm{FM}, 3,6 \\ \text { 7. } z x=z(y x) & \mathrm{AM} \\ \text { 8. } z(y x)=(z y) x & \mathrm{R} 2,7,8 \\ \text { 9. } z x=(z y) x & \mathrm{I} \\ \text { 10. } x=x & \mathrm{FM}, 2,10 \\ \text { 11. }(z y) x=0 x & \mathrm{R} 2,9,11 \\ \text { 12. } z x=0 x & \mathrm{~N} 1 \\ \text { 13. } 0 x=0 & \mathrm{R} 2,12,13 \\ \text { 14. } z x=0 & \end{array}$

These deductions are given for several reasons. In the first place, not only are these what one would expect to find in Boole's writings given what Boole himself says on pages 17 and 18 of $M A L$ where he describes his system but also deductions of this sort are strongly suggested to the modern reader of some of Boole's later works ([6], pp. 231-239). In the second place, they give some idea of what is possible using a system like the one that Boole described and they give some idea of the glaring omissions from Boole's system. In the third place, as will be seen below, they are useful in providing a sharp contrast with the way that Boole actually "established" the validity of the two Aristotelian syllogisms in question.

2.4 Solutions and consequences of equations In the next section we show that Boole repeatedly committed a fallacy involving taking a solution to an 
equation to be a consequence. In order to make the issues clear, this section is devoted to articulation of some distinctions which are widely presupposed in mathematical discourse but which, to the best of our knowledge, have never been spelled out in print. At the end of this section we explain how Boole's fallacy can be seen as a rather natural outcome of overly informal uses of the terms 'deduce' and 'imply' which were common in the mathematical literature of his day and which are relatively common even today.

The phrase "a solution to an equation" has been used in several senses, four of which are explicated in this section. For purposes of illustration we consider only equations in two variables $x$ and $y$ taking values in the domain $\mathbb{Z}$ of all integers. For simplicity we limit our explications to equations in two variables. Generalization to arbitrary equations is obvious.

In one sense, the ordered pairs $(0,0)$ and $(2,2)$ are the only solutions to the equation $x+y=x y$ and the ordered pairs $(1,3)$ and $(-1,3)$ are the only solutions to the equation $x^{2} y=3$. What is meant here, of course, is more fully expressed by saying, e.g., that -1 assigned to $x$ and 3 assigned to $y$ (together) form a solution to $x^{2} y=3$. The ordered pair locution presupposes a prior ordering of the variables: $(3,-1)$ is not a solution to $x^{2} y=3$ (unless $y$ is linked with the first member and $x$ with the second).

This sense of solution may be explicated by defining a solution to an equation to be an assignment (of objects in its domain to its variables) which satisfies the equation. In the case of $x+y=x y$, the assignments, $\alpha$ and $\beta$, are defined as follows:

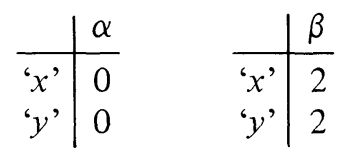

Let us call solutions in this sense root solutions because they involve what are often called the "roots of an equation". Let $p(x, y)$ and $q(x, y)$ be terms (e.g., polynomials) involving only the variables $x$ and $y$. When one asks for a root solution to the equation $p(x, y)=q(x, y)$, the variables are taken as names of "unknown objects" and the equation is regarded hypothetically as a true statement about those objects. The problem is to find out exactly which objects the statement is "true of".

In many cases, of course, an equation has a countably infinite number of root solutions. For example, every assignment $\gamma$ such that

$$
\left[\gamma^{6} x^{\prime}\right]^{2}+\left[\gamma^{6} y^{\prime}\right]^{2}=\left[\gamma^{6} z^{\prime}\right]^{2}
$$

is a root solution to $x^{2}+y^{2}=z^{2}$. Also, the root solutions to $x^{2}-y^{2}=0$ are the assignments $\gamma$ such that $\gamma^{6} x^{\prime}=\gamma^{6} y^{\prime}$ or $\gamma^{6} x^{\prime}=-\gamma^{6} y^{\prime}$.

Now the set of solutions $\gamma$ with $\gamma^{6} x^{\prime}=\gamma^{6} y$ ' can be "combined" into a (total) function, viz., $g(n)=n$. Likewise all solutions $\gamma$ with $\gamma^{6} x^{\prime}=-\gamma^{6} y^{\prime}$ ' can be combined into another function, viz., $h(n)=-n$. Each root solution to $x^{2}-$ $y^{2}=0$ is a "member" of exactly one of the two functions $g$ and $h$ but uncountably many other combinations are possible, e.g., the solutions $\gamma$ with $\gamma^{6} x^{\prime}=$ $\gamma^{\prime} y^{\prime}$ where $\gamma^{6} x^{\prime}$ is odd combine with the solutions $\gamma$ where $\gamma^{6} x^{\prime}=-\gamma^{6} y^{\prime}$ for $\gamma^{6} x^{\prime}$ even to form the function $j$ such that $j(n)=n$ for $n$ odd and $j(n)=-n$ for $n$ even. 
Notice that no such combining into total functions is possible with the solutions to $x+y=x y$ because there are not enough solutions, e.g., there is no solution assigning 1 to ' $x$ '.

In another sense of the term 'solution', one considers the equation not as a condition on objects but rather as a condition on functions. In this sense when one asks for a solution to $x+y=x y$ one is really asking for a (total) function $x(y)$ such that

$$
x(y)+y=x(y) y, \text { for each integer } y
$$

or, to put it more precisely, one seeks a function $f$ such that

$$
f(y)+y=f(y) y \text {, for each integer } y .
$$

In this sense $x+y=x y$ has no solutions and $x^{2}-y^{2}=0$ has an uncountably infinite number of solutions, two expressible by the equations $g(y)=y$ and $h(y)=-y$. In general we call a function $f$ a functional solution ("for $x$ as a function of $y$ ") to an equation $p(x, y)=q(x, y)$ iff for all $y, p(f(y), y)=$ $q(f(y), y)$.

In some cases, as we have seen, functional solutions can be expressed by equations. Sometimes mathematicians are not as explicit as possible in this regard. For example, instead of expressing one of the functional solutions to $x^{2}-y^{2}=0$ by " $h(y)=-y$ for all $y$ " this sometimes is expressed simply by the equation " $x=-y$ " (understood as " $x(y)=-y$, for all $y$ "). In either case the term on the right can be called the defining term of an expression of a functional solution.

This brings us to a third sense of 'solution' because sometimes the defining terms are called solutions. In this sense ' $y$ ' and ' $-y$ ' could be said to be solutions ("for $x$ as a function of $y$ ") to the equation $x^{2}-y^{2}=0$. These we call term solutions. Note that when a polynomial is said to be a solution to an equation the word "solution" is being used in the sense of a term solution.

Finally we note that equations are sometimes said to be solutions of equations. In particular, an equation expressing a functional solution is often itself called a solution to an equation. For example, $x=y$ is sometimes said to be a solution to $x^{2}-y^{2}=0$. For this sense we use the phrase equational solution: where $t(y)$ is a term involving only the variable $y$, the equation $x=t(y)$ is said to be an equational solution ("for $x$ as a function of $y$ ") to an equation when the function $f$ such that for all $y, f(y)=t(y)$, is a functional solution.

It is probably worth noting that there are equations which are called solutions in the literature which are not equational solutions in the above sense. For example, in Boole's book on differential equations ([5], p. 7) $x y=\sin x$ is referred to as "a solution" to the differential equation resulting from differentiating it with respect to $x$. Boole's "solution", of course, is equivalent to $y=x^{-1} \sin x$ which is, in our sense, an equational solution (for $y$ as a function of $x$ ) to the same equation.

Let $p(x, y)$ and $q(x, y)$ indicate terms (e.g., polynomials) involving the variables $x$ and $y$. The following principles summarize the above distinctions.

1. The assignment $\gamma$ is a root solution to an equation $p(x, y)=q(x, y)$ iff the sentence $p\left(\gamma^{6} x^{\prime}, \gamma^{\prime} y^{\prime}\right)=q\left(\gamma^{6} x^{\prime}, \gamma^{6} y^{\prime}\right)$ is true. 
2. The function $f$ is a functional solution to $p(x, y)=q(x, y)$ iff the sentence "For every $y, p(f(y), y)=q(f(y), y)$ " is true.

3. The term $t(y)$ is a term solution to $p(x, y)=q(x, y)$ iff the sentence "For every $y, p(t(y), y)=q(t(y), y)$ " is true.

4. The equation $x=t(y)$ is an equational solution to $p(x, y)=q(x, y)$ under the same conditions as in 3 , i.e., to verify that $x=t(y)$ is an equational solution it is sufficient to substitute $t(y)$ for $x$ in the equation and then to ascertain that the result is true for all values of $y$.

For example, to verify that $\mathrm{x}=-y$ is a solution to $x^{2}-y^{2}=0$ it is sufficient to ascertain that $(-y)^{2}-y^{2}=0$ is true for all values of $y$ in the domain of integers. This trivial point has special significance in interpreting Boole's procedures.

The root solutions are relevant when the problem is simply to find "the roots" of an equation and the other three sorts of solutions are relevant when the problem is "to describe one variable as a function of the others", which, from a foundational point of view, is the same as the problem of combining "roots" into a function in the strict sense. The last three sorts of solutions are very closely related but there is a way that the notion of a functional solution is opposed to the notions of term solution and equational solution. For example, there is an uncountably infinite number of functional solutions to $x^{2}-y^{2}=0$ but only a countable number of term solutions and equational solutions. But each term solution and, therefore, each equational solution can be regarded as an expression of a functional solution. We remind the reader that the above remarks apply literally only to equations in two variables $x$ and $y$, and that modifications are in order when other equations are considered. For example, $x=2$ can be said to be an equational solution to $x^{2}=4$ and there is no question of the equation having a functional solution.

Of special importance in this article are the equational solutions, and serious confusion is likely to occur unless it is noted that equations are, strictly speaking, ambiguous. They have one sort of meaning when used in problems about "unknown" objects (numbers, classes, etc.) and a completely different sort of meaning when presented as solutions to equations. For example, when $x=y+z$ is used in a problem about numbers it expresses an identity (of the number $x$ to the sum of $y$ and $z$ ) but when used to express a solution (e.g., to $x-z=y$ ) it is read as a definition of a function, i.e., as "for every $y$ and $z$, $x(y, z)=y+z$ ". In the problem ' $x$ ' is an "arbitrary name", in the solution ' $x$ ' is a "dependent variable", i.e., a disguised function symbol.

There are three important things to notice about the relationships between an equational solution and the equations that it solves. Here all equations are read as equations expressing identities. First, every equation is a consequence of each of its equational solutions. For example, $x^{2}-y^{2}=0$ is a consequence both of $x=y$ and of $x=-y$. Second, an equational solution is not necessarily a consequence of an equation which it solves. For example, $x=y$ does not follow from $x^{2}-y^{2}=0$, nor does $x=-y$ follow. In this case, of course, the disjunction of the two solutions does follow, but the disjunction of two equational solutions is not itself an equational solution. This implies the third point, viz., that a consequence of an equation is not necessarily a solution. 
It is clear then that it would be a mistake to infer that an equation is a consequence given only that it is a solution. Below we explain that Boole made this mistake, but here we briefly indicate how this mistake might be traced to overly informal uses of the terms "deduce" and "imply".

In former times (Boole [5], p. 8) and to some extent even recently (Langer [19], p. 359), mathematicians used the word "deduction" vaguely to indicate any process by which a solution was extracted from an equation regardless of whether the process was limited to logical inference. Langer defines a solution to a differential equation in $x$ and $y$ to be any equation in $x$ and $y$ (involving no derivatives) which is deducible from the equation. If one speaks of solutions as being deduced from equations then it is more or less natural to refer to solutions as consequences of those equations.

Moreover, as Boole himself states ([5], p. 6), there is a sense in which a solution is implicit in an equation. An equation describing a physical process does, in some sense, imply that the "quantities" involved are related in accord with the solutions. But unless one is clear that this is not necessarily logical implication of a solution by the equation, one is liable to mistake solutions for consequences.

2.5 Boole on conversion of propositions Two pages after presenting his deductive system Boole introduces his notorious " $v$ ". The symbol $v$ is supposed to indicate a nonempty class, so that $v=x y$ expresses "Some $X \mathrm{~s}$ are $Y \mathrm{~s}$ " $(M A L$, p. 21). There is considerable difficulty in deciphering Boole's intentions in regard to $v$ (Dummett [15], p. 205; van Evra [23], p. 370), and we will not make an attempt here. For our purposes it is enough to know that Boole believed "Some $X \mathrm{~s}$ are $Y$ " to be expressible as $v=x y$, as $v x=v y$, and as $v x(1-y)=0$ and that he believed "Some $X$ s are not $Y \mathrm{~s}$ " to be expressible as $v=x(1-y)$, as $v x=x(1-y)$ and as $v x y=0$. It is possible to make a certain amount of sense of this if, as Boole suggests, one reads ' $v x$ ' (and in some cases ' $v$ ' alone), as "some $X \mathrm{~s}$ ". For example, $v x=v y$ is read (by Boole) "Some $X$ s are some $Y \mathrm{~s}$ ", and $v=x y$ is read sometimes as "Some $X \mathrm{~s}$ are $Y \mathrm{~s}$ " and sometimes as "Some $Y \mathrm{~S}$ are $X \mathrm{~s}$ " $(M A L$, p. 21). In addition Boole reads $v(1-y)$ as "Some not-Ys" (MAL, p. 25).

Boole gives no special rules or axioms for making deductions involving the symbol $v$. He does however claim to have derived, e.g., "Some $Y \mathrm{~s}$ are $X \mathrm{~s}$ " from "All $X \mathrm{~s}$ are $Y \mathrm{~s}$," and Boole's "derivations" of the Aristotelian conversions involved him in the fallacy of taking a solution to an equation as a consequence, as is shown by the following passage (MAL, pp. 26-28).

The primary canonical forms already determined for the expression of Propositions, are

$\begin{array}{lll}\text { All } X \mathrm{~s} \text { are } Y \mathrm{~s}, & x(1-y)=0 & \mathrm{~A} \\ \text { No } X \mathrm{~s} \text { are } Y \mathrm{~s}, & x y=0 & \mathrm{E} \\ \text { Some } X \mathrm{~s} \text { are } Y \mathrm{~s} & v=x y & \mathrm{I} \\ \text { Some } X \mathrm{~s} \text { are not- } Y \mathrm{~s} & v=x(1-y) & 0 .\end{array}$

The equations $\mathrm{A}$ and $\mathrm{E}$, written in the forms

$$
\begin{aligned}
& (1-y) x=0 \\
& y x=0
\end{aligned}
$$


give on solution the respective forms

$$
\begin{aligned}
& x=v y \\
& x=v(1-y)
\end{aligned}
$$

the correctness of which may be shown by substituting these values of $x$ in the equations to which they belong, and observing that those equations are satisfied quite independently of the nature of the symbol $v$. The first solution may be interpreted into

\section{Some $Y$ s are $X \mathrm{~s}$}

and the second into

Some not- $Y$ s are $X$ s.

From which it appears that universal affirmative and universal negative Propositions are convertible by limitation, or, as it has been termed, per accidens.

There should be no mistake about our claim that Boole's sole ground for asserting that $x=v y$ follows from $(1-y) x=0$ is given above. This is so despite the fact that it should have been obvious to Boole that $x=0$ is also a solution and that if $x=0$ and $y$ is any nonempty set then the equation is true but the solution $x=v y$ is false (for $v$ any set not disjoint with $y$ ).

One should also be clear about the fact that $x=v y$ is not deducible from $(1-y) x=0$ using the rules that Boole explicitly states. The reason is simply that Boole's explicitly stated rules are all satisfied when interpreted as in ordinary class algebra.

For convenience we use the phrases "solutions fallacy" and "the fallacy of solutions" to indicate the inference of a solution of an equation from the equation itself, when the solution doesn't follow. Strictly speaking it is always a fallacy to infer a solution from an equation on the ground that it is a solution, even when the solution actually follows. But we use the term in the narrower sense which does not have reference to the ground of the inference. ${ }^{12}$

It is clear enough that Boole is guilty of the solutions fallacy in the above case. It has not been shown, however, that Boole believed that each equation used to express a particular proposition ("Some $X \mathrm{~s}$ are $Y \mathrm{~s}$ " or "Some $X \mathrm{~s}$ are not- $Y \mathrm{~s}$ ") is deducible in his system from each equation used to express any implying general proposition ("All $X \mathrm{~s}$ are $Y \mathrm{~s}$ " or "No $X \mathrm{~s}$ are $Y \mathrm{~s}$ "). This question is a bit more complicated than it appears because Boole permitted a single given proposition to be expressed by as many as three different equations. But after presenting his deductive system and correlating the equations with propositions he states (MAL, p. 23):

... all the equations by which particular truths are expressed are deducible from any one general equation expressing any one general proposition from which those particular propositions are necessary deductions. (emphasis ours)

Of course Boole is using the term "necessary deduction" to indicate either "necessary consequence" or "necessary deduction of the Aristotelian system". Moreover, at the very beginning of the first chapter of $M A L$ (p. 6) Boole says that in the system to be presented the laws of conversion and of syllogisms will be deducible. 
2.6 Boole's derivations of the syllogisms On pages 31 through 47 of $M A L$ Boole gives his treatment of the syllogisms. From earlier pages one might expect that Boole would use more or less straightforward deductions except in the cases where Aristotle had deduced a particular conclusion from universal premises. In these cases one would expect to find the solutions fallacy. In turning to the text one does indeed find the solutions fallacy bridging the gap between universal premises and particular conclusions. However, not even in the other cases does one find straightforward deductions.

In order to understand Boole's method of dealing with syllogisms one must recall that, from two equations in three variables, it is sometimes possible to deduce a third equation involving only two of the variables. For example $x+y=z$ and $5 x+2 y=3 z$ imply $3 x=z$. Boole knew that in certain cases it is possible to use a general rule for "eliminating" $y$ from the equations. On page 32 he notes that the following elimination scheme is valid in arithmetic.

E15

$$
\begin{gathered}
a y+b=0 \\
a^{\prime} y+b^{\prime}=0 \\
? a b^{\prime}-a^{\prime} b=0 .
\end{gathered}
$$

To apply this to the above case the equations must be rewritten in the required forms:

$$
\begin{array}{lll}
x+y=z & \text { is rewritten } & 1 y+(x-z)=0 \\
5 x+2 y=3 z & \text { is rewritten } & 2 y+(5 x-3 z)=0 .
\end{array}
$$

Then applying the elimination scheme one has:

$$
1(5 x-3 z)-2(x-z)=0 .
$$

From here one can deduce $3 x=z$.

Boole also seems to have noticed that E15 is also valid in class logic.

The above elimination scheme E15, which Boole referred to as "(15)", is the only such scheme symbolically stated in $M A L$ but it is not the only one mentioned. On page 34, Boole writes:

A convenient mode of effecting the elimination is to write the equations of the premises so that $y$ shall appear only as a factor of one member in the first equation, and only as a factor of the opposite member in the second equation, and then to multiply the equations, omitting the $y$.

His example (MAL, p. 35) is quoted below.

$$
\begin{array}{rr}
\text { All } X \text { s are } Y \mathrm{~s} \quad x(1-y)=0 & \text { or } x=x y \\
\text { No } Z \text { s are } Y \mathrm{~s} & \quad \therefore \begin{array}{r}
z y=0 \\
z x=0
\end{array} \\
& \therefore \text { No } Z \text { are } X \mathrm{~s} .
\end{array}
$$

Boole evidently intends the premises to be written $b=a y$ and $a^{\prime} y=b^{\prime}$. There are two forms of the conclusion compatible both with his words and with the example, viz., $a^{\prime} b=a b^{\prime}$ and $a a^{\prime}=b b^{\prime}$. The first is valid in general but the second is not [e.g., take $a=a^{\prime}=1$ ]. Thus we represent Boole's second elimination scheme as follows. 
E $\quad b=a y$

$a^{\prime} y=b^{\prime}$

$? a^{\prime} b=a b^{\prime}$

Boole gives no derivations for either of these two schemes. However both are easily seen to be valid.

Deduction of $\mathrm{E}$
1. $b=a y$
2. $a^{\prime} y=b^{\prime}$
? $a^{\prime} b=a b^{\prime}$
3. $a^{\prime} b=a^{\prime}(a y)$
4. $a\left(a^{\prime} y\right)=a b^{\prime}$
5. $a^{\prime}(a y)=\left(a^{\prime} a\right) y$
6. $a\left(a^{\prime} y\right)=\left(a a^{\prime}\right) y$
7. $a^{\prime} b=\left(a^{\prime} a\right) y$
8. $\left(a a^{\prime}\right) y=a b^{\prime}$
9. $a a^{\prime}=a^{\prime} a$
10. $\left(a a^{\prime}\right) y=\left(a^{\prime} a\right) y$
11. $a^{\prime} b=\left(a a^{\prime}\right) y$
12. $a^{\prime} b=a b^{\prime}$
QED

multiplying $a^{\prime}$ times (1)

multiplying $a$ times (2) associativity associativity things equal to the same $(5,3)$ things equal to the same $(6,4)$ commutativity multiplying (9) times $y$ things equal to the same $(7,10)$ things equal to the same $(8,11)$

A derivation of E15 is an elaborate triviality not worth citing in detail. One way to proceed is to first derive $b^{\prime}=0$ from $a^{\prime} y+b^{\prime}=0$ and then from $b^{\prime}=0$ derive $a b^{\prime}-a^{\prime} b=0$. This reasoning shows that Boole's E15 is very weak and that it is a special case of the following.

SE

$$
\begin{gathered}
a y+b=0 \\
a^{\prime} y+b^{\prime}=0 \\
? z b^{\prime}-w=0
\end{gathered}
$$

The point to notice is that the conclusions, both of E15 and of SE, follow from the second premise $\left(a^{\prime} y+b^{\prime}=0\right)$ alone. Moreover, the only thing that is relevant to the conclusion is the fact that the second premise implies $b^{\prime}=0$. Thus whenever Boole uses E15 he is using only the second premise. ${ }^{13}$

Boole believed that elimination schemes provide an essential part of derivations of syllogistic conclusions. Just prior to stating E15 he wrote:

The equation by which we express any Proposition concerning the classes $X$ and $Y$ is an equation between the symbols $x$ and $y$, and the equation by which we express any Proposition concerning the classes $Y$ and $Z$ is an equation between the symbols $y$ and $z$. If from two such equations we eliminate $y$, the result, if it do not vanish, will be an equation between $x$ and $z$, and will be interpretable into a proposition concerning the classes $X$ and $Z$. And it will then constitute the third member, or Conclusion, of a Syllogism of which the two given Propositions are the premises. (MAL, pp. 31, 32, italics added)

Shortly after this statement he explains the qualification "if it do not vanish". Notice that if $b$ and $b^{\prime}$ are both 0 then the elimination scheme yields, in effect, $0=0$. Boole explains that in such cases one does not apply a scheme 
to the two equations directly but one must first solve one of them. And he repeats his observation that $y=v(1-x)$ is a solution to $x y=0$ and that $y=v x$ is a solution to $(1-x) y=0$. (He seems to think that these are the only solutions, but for the present this can be ignored.)

We quote in full Boole's treatment of the first Aristotelian syllogism (MAL, p. 76).

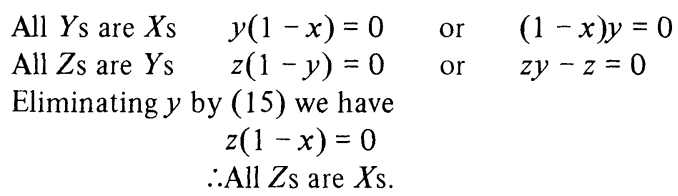

That Boole did not correctly apply E15 to get his conclusion will become clear presently. What is immediately clear, however, is that Boole thinks that he used E15 to deduce $z(1-x)=0$ from $(1-x) y=0$ and $z y-z=0$. But this is a fallacy because $z y-z=0$ is a logical truth: $z y$ must be a subset of $z$, and the complement of $z$ with respect to one of its own subsets is necessarily 0 . In other words, no matter what classes $y$ and $z$ may be, $z y-z=0$ is true. Thus $z(1-x)=0$ is being deduced from $(1-x) y=0$, which does not imply it, of course (take $x=y=0$ and $z=1$ ).

This and other passages suggest that Boole may not have been using "-_" to indicate relative complement. But in $M A L$ he never explicitly states what he takes "-" to indicate. Compare ([15], p. 205). In later works (e.g., [4], p. 33) he takes ".." to indicate a certain partial function. Regardless of whether Boole is using "-" to indicate a partial function as in Laws of Thought, in the course of "applying" E15, there are other fallacies involved in the above quoted passage. ${ }^{14}$

The inescapable conclusion is that Boole is not using E15 as he says he is. Moreover, the important point is not that there is a gap between what he claims to be doing and what he is doing, but rather that no care has been taken to refer his derivations to the deductive system that he has set forth several pages earlier.

Boole's second example was quoted in full above in connection with the discussion of elimination scheme E. Likewise in that example the connection, if any, with the deductive system is not referred to.

In the next four examples $(M A L$, pp. 35, 36) Boole derives particular conclusions from universal premises. In each of these cases he uses the fallacy of solutions to get intermediate particular propositions and then he applies elimination scheme E. For example, in the first of these he infers the solution $y=v x$ from $y(1-x)=0$, failing to notice that the solution is not a consequence $(y(1-x)=0$ does not imply that $y$ is nonempty).

In the final three (MAL, p. 37) of his nine examples he deduces particular conclusions from a pair of premises each including a particular premise. In these cases, of course, no fallacy is needed to get a particular conclusion. Nevertheless in the second of these his second step seems, in effect, to deduce "Some $Y \mathrm{~s}$ are not $Z \mathrm{~s}$ " from "Some $Z \mathrm{~s}$ are not $Y \mathrm{~s}$ " (e.g., "Some dogs are not animals" from "Some animals are not dogs"). See the example on page 632. 
In order to summarize Boole's treatment of the syllogisms we make the following abbreviations.

$\begin{array}{ll}A x y & \text { All } X \mathrm{~s} \text { are } Y \mathrm{~s} \\ N x y & \text { No } X \mathrm{~s} \text { are } Y \mathrm{~s} \\ S x y & \text { Some } X \mathrm{~s} \text { are } Y_{\mathrm{s}} \\ S x(1-y) & \text { Some } X \mathrm{~s} \text { are not- } Y \mathrm{~s} \\ S(1-x) y & \text { Some not- } X \mathrm{~s} \text { are } Y \mathrm{~s} \\ S(1-x)(1-y) & \text { Some not- } X \mathrm{~s} \text { are not- } Y \mathrm{~s} .\end{array}$

1. (1) $A y x$

(2) $A z y$

? $A z x$

2. (1) $A x y$

(2) $N z y$

? $N z x$

3. (1) $A y x$

(2) $N z y$

? $S x(1-z)$

4. (1) $A y x$

(2) $N z y$

? $S(1-z) x$

5. (1) $A y x$

(2) $A y z$

? $S x z$

On page 34, E15 is misapplied to inferences from the premises.

On page $35, \mathrm{E}$ is applied to inferences from the premises.

On page $35, \mathrm{E}$ is applied to a solution of (1) taken together with an inference from (2).

On page $36, \mathrm{E}$ is applied to (1) taken together with a solution of (2).

On page $36, \mathrm{E}$ is applied to a solution of (1) taken together with an inference from (2).

On page $36, \mathrm{E}$ is applied to an inference from (1)

6. (1) $N y x$

(2) $N z y$

taken together with a solution of (2).

? $S(1-z)(1-x)$

7. (1) $A y x$

On page $37, \mathrm{E}$ is applied to (1) and (2).

(2) $S z y$

? $S z x$

8. (1) $A x y$

(2) $S z(1-y)$

? $S z(1-x)$

On page $37, \mathrm{E}$ is applied to inferences from (1) and (2).

9. (1) $S y(1-x)$

(2) $\mathrm{Nzy}$

On page $37, \mathrm{E}$ is applied to (1) and an inference

? $S(1-x)(1-z)$

In his treatment of the syllogism Boole does not explicitly employ the deductive system which he has described on pages 17 and 18. Instead he treats syllogistic inference as a case of eliminating a variable from two equations. To effect this he gives two elimination schemes; one, E15, is stated symbolically and the other, E, is stated ambiguously. The one which is stated symbolically is applied once, and then incorrectly. The one which is not stated symbolically is used eight times. In one case it seems to be used in conjunction with an elementary conversion fallacy, and in four other cases it is used in conjunction with the fallacy of solutions. Since E was not deduced, none of the derivations are proper deductions.

If it is not discouraging enough that the fallacy of solutions occurs in four 
of his nine examples, in the discussion (MAL, p. 42) following the examples he says that it would have been allowable to make the same step in the other cases as well. We quote.

We have found it, in a certain class of cases, to be necessary to replace the two equations expressive of universal Propositions by their solutions; and it may be proper to remark that it would have been allowable in all instances to have done this ...

The inattention to logical detail exhibited in Boole's derivation of the syllogisms is clear enough so that further comment is not needed. It is remarkable, however, that Boole had the idea of a sound, equational deductive system but that he made no attempt to develop it or to apply it. It is clear that Boole's fallacious derivations of the syllogisms drawing particular conclusions from universal premises are all based on the fallacy of solutions. But one should not overlook the fact that other fallacies are also present.

3 Boole on establishing invalidity If a deductive system is "strong enough", i.e., if it contains a deduction of the conclusion from the premises whenever the conclusion is implied by the premises then it is said to be complete. This could have been what Boole had in mind by his term "sufficient". But in view of Boole's failure to clearly distinguish consequences from solutions he could have been thinking of his system as a calculus, i.e., as a method of deriving solutions to equations (and sets of equations). It would be natural to call a calculus "complete" if it contains a derivation of a given equation from a given set of equations whenever the given equation is a solution to the set of equations.

Of course, one must also take account of the possibility of having "unsound deductions", i.e., deductions which permit inference from given premises of conclusions which do not follow. In such a case one says informally that the deductive system is "too strong". If a deductive system is not "too strong", i.e., if it permits deduction of a conclusion from premises only in those cases where the argument is valid, then it is said to be sound. For a deductive system to be complete and sound is for it to be "just strong enough". In this case one can use it to deduce each valid argument but one cannot use it to deduce any invalid arguments. It apparently never occurred to Boole to wonder whether his system (or his method) was unsound. Indeed, he paid only the most casual notice to the concept of invalidity.

As indicated above, the usual criterion of invalidity is refutability by a counterargument. One knows that "All men are happy and Joe is happy" does not imply "Joe is a man" because one knows that "All dogs are animals and Felix is an animal" is true whereas "Felix is a dog" is known to be false. Boole notes the invalidity of many arguments but he never uses the method of refutation by counterargument (even implicitly) to establish his observations. In his discussion of conversions (MAL, pp. 26-30) he does not discuss, or even mention, invalid conversions such as "All $X \mathrm{~s}$ are $Y \mathrm{~s}$ " with "All $Y \mathrm{~s}$ are $X \mathrm{~s}$ ". Moreover in his discussion of syllogisms, although invalid arguments are mentioned, there is not one counterargument to be found in the entire fifteen pages ( $M A L$, pp. 31-47). 
As mentioned above, the method of refutation by counterargument is virtually implied by the so-called principle of form, viz., that arguments are valid or not in virtue of form, i.e., that two arguments in the same form are both valid or both invalid. Boole indicates some awareness of form as opposed to content in $M A L$. And in later works ([6], pp. 216, 217) Boole emphasizes the fact that validity does not depend on the particular meanings of the terms employed but he never seems to see this fact as leading to a method of refutation. It is as if establishing invalidity by counterargument has never occurred to him.

It might be suspected that refutation by counterargument was somehow out of favor in the time during which Boole wrote. But this suspicion is neither confirmed nor put to rest by consulting the several works on logic written in the British Isles just prior to Boole's work. For example, Whately's Elements of Logic [24], which was supposedly the most popular logic book of the period and which was quoted or referred to four times by Boole $(M A L, \mathrm{pp} .7,18$, 20,28 ), uses the method of counterarguments repeatedly. But there is no discussion of it and there is no systematic use of it that would enable one to infer that a principle was being tacitly appealed to. Some of Whately's counterarguments are quoted below.

1. All birds are animals.

?All animals are birds.

2. Some animals are beasts.

Some animals are birds.

?Some birds are beasts.

3. Some animals are sagacious.

Some beasts are not sagacious.

?Some beasts are not animals.

The method of refutation by counterargument is conspicuously absent from Mill's famous System of Logic [20] (originally published in 1843 and referred to in $M A L$, p. 2). Despite the fact that Mill seems to be unaware, not only of the traditional criterion of invalidity but also of the traditional deductive criterion of validity, it must be admitted that he seems to refer the reader elsewhere for these ideas. After displaying the traditional valid two premise syllogisms, he writes:

The reason why syllogisms ... are legitimate, that is, why if the premises are true the conclusion must inevitably be so, and why this is not the case in any other possible combination . . . of propositions ... may be presumed to have [been] . . . learned from the common school books of syllogistic logic .... The reader may . . . be referred . . . to ... Whately's Elements of Logic, where he will find stated with philosophical precision, and explained with remarkable perspicuity, the whole of the common doctrine of the syllogism. ([20], p. 191)

In many respects DeMorgan's Formal Logic [14], published in 1847, the same year as $M A L$, is a breath of fresh air compared to Whately, Mill, and Boole. DeMorgan is much more sensitive both to language and to the conventions which had become traditional in logic but which deviated from those 
common in ordinary discourse. In addition he is much more systematic and his attention to detail is what one would expect from a mathematician. However there is nothing about organizing deduction into a system and, although the method of refutation by counterargument seems just below the surface in many places, one could not argue that DeMorgan was consciously aware of it as a method.

\subsection{Boole's criterion of invalidity In order to grasp Boole's treatment} of invalidity it is useful to recall a few elementary ideas from analytic geometry. An equation in three variables $f(x, y, z)=g(x, y, z)$ describes a set of points in three-dimensional space. For example, $z=x+y$ describes the plane containing the three points $(0,0,0),(1,0,1)$, and $(0,1,1)$. This plane cuts each plane $(z=$ constant) parallel to the $x y$-plane in a line $y=z-x$ (which for $z=0$ is the line $y=-x$ ). For another example, $z^{2}=x^{2}+y^{2}$ describes the cone obtained by rotating the line $z=y$ about the $z$-axis. This cone cuts each plane parallel to the $x y$-plane in a circle whose radius $\left(x^{2}+y^{2}\right)^{1 / 2}$ is the distance $|z|$ of the given plane from the $x y$-plane.

The set of points described by a law of arithmetic, e.g., $x \cdot(y \cdot z)=(x \cdot y) \cdot z$, is the set of all points in the space. An equation which is "universally false", e.g., $x \cdot(y \cdot z)+1=(x \cdot y) \cdot z$, describes the null set of points. And the set of points described by several equations taken together is the set of points common to the sets described by the individual equations. For example the set of points described by $z=x+y$ and $z^{2}=x^{2}+y^{2}$ taken together contains two points $(0, z, z)$ and $(z, 0, z)$ in each plane $(z=$ constant) parallel to the $x y$-plane and thus consists of the two lines $z=x$ (in the plane $y=0$ ) and $z=y$ (in the plane $x=0$ ).

If an equation $c$ is implied by a set $P$ of equations then the set of points described by the members of $P$ (taken together) is a subset of the set of points described by $c$.

The equation $0=0$ is implied by every set of arithmetic equations: $p=q$ implies $p-p=q-q$ or $0=0$. Moreover, $0=0$ is equivalent to $(x-x)+(y-y)=$ $(z-z)$, so $0=0$ can be regarded as describing the whole space.

The situation in Boole's class algebra is exactly analogous. An equation in three variables describes a set of triples of classes. Several equations taken together describe the triples of classes common to the sets of triples described by the individual equations. The set of triples described by a consequence of an equation contains as a subset the class of triples described by the equation. Each Boolean law, including $0=0$, describes the set of all triples of classes. And $0=0$ is implied by each and every set of equations.

The important point to bear in mind here, both with respect to arithmetic as well as in Boole's algebra, is that nothing whatever can be concluded about a set of equations from the fact that $0=0$ has been deduced from it. The above discussion was designed to put this point in a context that would make it completely clear and unproblematic.

Boole first makes note of invalidity on page 35 where he makes the assertion that there is no inference possible from "All $X \mathrm{~s}$ are $Y \mathrm{~s}$ " and "All $Z \mathrm{~s}$ are $Y \mathrm{~s}$ ". Of course, no valid Aristotelian syllogism has these as premises but clearly these two premises imply "All $(X$ or $Z$ )s are $Y$ s", "All ( $X$ and $Z$ )s are $Y \mathrm{~s}$ ", "All 
$X \mathrm{~s}$ except ${ }^{15} Z \mathrm{~s}$ are $Y \mathrm{~s}$ ", and so on, all of which are easily representable in Boole's notation. One must assume that Boole intended to restrict himself to the Aristotelian cases here even though he considers valid but non-Aristotelian inferences on the very next page.

What is somewhat baffling is that the only ground that Boole gives for asserting that no inference is possible in the above case is that $0=0$ is deducible from the result of applying elimination scheme $\mathrm{E}$ to the premises $x=x y$ and $z y=z$. But he doesn't state that that is the ground.

On page 38 he does something similar with each of two pairs of Aristotelian premises which do not yield syllogistic conclusions. But in these cases, instead of deducing $0=0$ from the result of applying the elimination scheme $E$, he deduces $0=0$ from the result of applying $E$ taken together with what he calls "the auxiliary equation" for the second premise. To understand what he has in mind by "auxiliary equation" one should recall that $v$, the "auxiliary symbol", is supposed to mean "Some $X \mathrm{~s}$ " in certain contexts. In these contexts the auxiliary equation is $v(1-x)=0$. In contexts where $v$ is supposed to mean "Some not- $X \mathrm{~s}$ " the auxiliary equation is $v x=0$ (see $M A L$, pp. 24, 25). Although Boole does not explicitly say so, it is clear that the auxiliary equation is "attached" to a given equation when used to express a given proposition and it is clear that the auxiliary equation states that $v$ is a subset of the class that Boole's usage takes it to be a subset of. According to Boole's usage, e.g., "All $Y \mathrm{~s}$ are $X \mathrm{~s}$ " is expressed by $y=v x$ with $v(1-x)=0$ as auxiliary. Of course, it would have been much clearer to say that "All $X \mathrm{~s}$ are $Y \mathrm{~s}$ " is expressed by the pair of equations $y=v x$ and $v(1-x)=0$.

On the basis of these three examples one might suppose that Boole thinks that the deduction of $0=0$ from the equations of premises (or from their equations taken together with their auxiliary equations) establishes that no Aristotelian conclusion follows. And this supposition is somewhat supported by Boole's own words after the third example.

Indeed, in every case in this class, in which no inference is possible, the result of elimination is reducible to the form $0=0$. Examples therefore need not be multiplied. (MAL, p. 38)

One should be clear about the fact that by "reducible to the form $0=0$ " Boole could not have meant "equivalent to $0=0$ " because in the example immediately preceding his quoted remark the result of elimination is $v x=v x z$ (and the auxiliary equations are $v(1-y)=0$ and $v(1-z)=0$ ).

On page 38 he considers his final class of "unlawful cases" which he divides in two. The first division is "when the result of elimination is reducible by auxiliary equations to the form $0=0$ ". The second is "when the result of elimination is not reducible by auxiliary equations to the form $0=0$ ". The first division is compatible with our supposition. His example of the latter division is the following, quoted in full (MAL, p. 39). See bottom of page 627 above.

$$
\begin{array}{lll}
\text { Some } Y \mathrm{~s} \text { are } X \mathrm{~s} & v y=v x & v y=v x \\
\text { Some } Z \mathrm{~s} \text { are not } Y \mathrm{~s} & v^{\prime} z=v^{\prime}(1-y) & \frac{v^{\prime}(1-z)=v^{\prime} y}{v v^{\prime}(1-z)=v v^{\prime} x}
\end{array}
$$


Now the auxiliary equations being

$$
v(1-x)=0 \quad v^{\prime}(1-z)=0
$$

the above reduces to $v v^{\prime}=0$. It is to this form that all similar cases are reducible. Its interpretation is that the classes $v$ and $v^{\prime}$ have no common member, as is indeed evident.

It is not at all clear why Boole thinks that this example is different from the others. Given the auxiliary equation $v^{\prime}(1-z)=0$, it follows that the left side, $v v^{\prime}(1-z)$, of the result of elimination is 0 . Thus the right side is 0 . Thus $0=0$ follows.

However the rest of the page in question, the next page, and the following page $(M A L$, pp. 39-41) make it plain that Boole believes that the last example is different.

In the whole of $M A L$ there is nothing on invalidity except the material reviewed above taken from pages 35 through 41 and some scattered remarks on pages 44 through 47 . There is not one counterargument in the entire book. No criterion of invalidity, correct or incorrect, is explicitly offered as such. And the only test which comes close to being used as such a criterion is deducibility of $0=0$ from the equations and auxiliary equations of the premises. But this has been seen to apply to every set of premises and thus does not serve to distinguish any argument from any other.

4 Conclusions concerning $M A L \quad$ Boole had an idea of a deductive system which, if developed, would have been superior to the system handed down by Aristotle. In the first place it permitted the use of logical axioms (instead of being restricted to rules of inference). In the second place, it could be used in connection with negative terms and with conjunctions and disjunctions of terms. ${ }^{16}$

But Boole did not seem to realize the importance of the ideas. He omitted many obvious axioms. He noticed two "derived rules" (E15 and E) but he gave no derivations of them. He did not give a single application of the system, not even easy and obvious ones such as derivations of the easiest of the Aristotelian syllogisms. In deriving the syllogisms using other methods (involving the "derived rules") fallacies have been noted in six of the nine examples. In four cases, the fallacy of solutions is involved. In a comment following the examples, Boole thinks that the fallacious move involving solutions is allowable in all cases. (The syllogisms drawing particular conclusions from universal premises are all obtained via the fallacy of solutions.)

The fact that Boole did not notice his fallacies may be partly explained by the fact that he was oblivious to the need for a criterion of invalidity. He mentions invalidity only within the space of twelve pages ( $M A L$, pp. 35-47). When he mentions the goals of logic he usually mentions the generation of valid inferences-never the identification and classification of invalid ones. He gives no criterion of invalidity and the test that he does seem to employ is absurdly inadequate.

Boole's standing in the history of logic does not, of course, rest on his achievements in The Mathematical Analysis of Logic, the work here being 
discussed. ${ }^{17}$ And it would not be fair to Boole to compare his understanding of logical methodology or his attention to logical detail,${ }^{18}$ as represented by $M A L$, to that of Aristotle as represented by Book I of Prior Analytics. It is worth pointing out, however, that Aristotle clearly stated his deductive criterion of validity and applied it with meticulous accuracy in twenty-odd deductions. Perhaps because Aristotle did so many examples, he managed to discover and state enough rules to have a complete deductive system (Corcoran [10]). It is also worth noting that although Aristotle did not state his counterargument criterion of invalidity, he applied it with precision and ingenious conciseness to over one hundred cases, making it possible to infer with considerable certainty what the criterion was.

Aristotle's work included the following experiment. He considered a rather simple class of almost two hundred arguments supplying a deduction for each valid case and supplying a counterargument for each invalid case. Of course, the arguments considered by Aristotle were syllogisms containing exactly one or exactly two premises. Boole would have discovered a complete set of axioms had he run an analogous experiment on the equations between the following terms (where $k=+, \cdot$ or - ): $0,1, x, x k y, x k(y k z),(x k y) k z$, $(x k y) k(x k z)$ (a total of 51 terms or 2601 equations). What we have in mind, of course, is supplying a deduction for each logical truth and a counterexample (in 0 and 1) for each of the others. We can excuse Boole from conducting the whole of such a huge and repetitious exercise. But whether Boole should be excused for running no such experiment is a matter to be judged by those with a greater appreciation for the state of logic and mathematics of the time.

Although we hereby register our doubts, we have no adequate ground for disagreement with Dummett [15] who wrote:

There can be no doubt that Boole deserves great credit for what he achieved, in the sense that in those historical circumstances what he did must have been very difficult to do.

\section{NOTES}

1. This interpretation of Aristotle's logical system was discovered independently by Smiley [21] and Corcoran [10]. Some related ideas were discovered earlier by Bacon [2].

2. The intended interpretation of the language in which the counterargument to $(P, c)$ is stated is, of course, a countermodel for $(P, c)$. Moreover, reinterpreting $(P, c)$ in one of its known countermodels produces a counterargument. Thus having a known countermodel and having a counterargument are the same thing. Boole, of course, uses the traditional terms 'argument', 'valid', 'train of reasoning', etc., and he has the idea of different interpretations (or models) of his formal language, but he does not introduce a special term for them.

3. It is very likely that Boole sometimes thought of + in $M A L$ as union. In Laws of Thought he explicitly takes + to be union on disjoint sets and undefined on overlapping sets, but such seems not to be the case in $M A L$. For example, in $M A L$, p. 58 , he speaks of adding the condition of disjointness in a situation where disjointness could be inferred were + partial. In this connection, and for other reasons as well, it is important to notice (1) that Boole never did interpret his operation symbols as truth-functions and (2) that in $M A L$ Boole never conceived of logic except as class logic. Propositions that 
are now treated by propositional logic were treated by Boole as equations between classes in a section called "Of Hypotheticals". There the elective symbol $x$ corresponding to the proposition $X$ indicates the class of "conceivable cases" in which $X$ is true. Thus $x+y$ is a term (not a sentence), it indicates a class of "cases" (not a truth value), and, so, + must be an operation on classes (not a truth-function) (MAL, pp. 48ff). References to Boole's "Theory of truth-functions" (e.g., Kneale and Kneale, [18], p. 420) are at best misleading. And reference to $x+y$ as expressing the truth-value of the exclusive disjunction of $X$ and $Y$ (e.g., [18], p. 413) is wrong for two reasons. First, it does not express a truth-value. Second, if + is union then $x+x=x$ so $x+y$ corresponds to the nonexclusive disjunction and if + is partial union then (for $x \neq 0$ ) $x+x$ is undefined so $x+y$ does not correspond to exclusive disjunction.

Boole introduces the minus sign $(M A L$, p. 20$)$ in the context $1-x$ and he uniformly uses it this way (as a binary function symbol in the expression of the nonrelative complement, which is a unary function, of course) for twelve pages $(M A L$, p. 32) where he first uses it without 1 in the context $a b^{\prime}-a^{\prime} b$. Then (MAL, p. 43), without comment, he uses it as a unary function symbol in the term $-v$. Thereafter he uses it as a binary function symbol and as a unary function symbol (e.g., $M A L$, pp. 46, 55).

4. This quotation is taken out of context ( $M A L$, p. 10), but it is clear from the broader context that it expresses Boole's view. Corroborating passages are also present (e.g., [6], pp. 17, 217, 218).

5. The distinction between logical axioms ("laws of judgment") and rules of inference ("laws of reasoning") is particularly crisp in $M A L$ because there is no "if . . then" in the object language. (This point is missed by Kneale and Kneale ([18], p. 412).) The logical axioms are stated using symbolic formulas and the rules of inference are given in English. Since "conducting an argument" ([6], p. 237) is clearly "reasoning" as opposed to "judgment", Boole feels it necessary to emphasize the necessity of using "laws of judgment" in deductions ([6], pp. 144, 237, 238). There are some passages which might appear to a modern reader to be attempts to articulate the axiom/rule distinction (e.g., [6], p. 25). But because the distinction is unproblematic for Boole, other interpretations are more likely.

6. Complementation should not be confused with negation. Not only did Boole's language lack negation but it also lacked the ability to express negations. For example, there is no Boolean formula involving $x$ and $y$ which is true if and only if $x$ and $y$ are distinct. Thus $x \neq y$ cannot be expressed. In a later work ([6], p. 128, fn) Boole explicitly considers "All $X \mathrm{~s}$ are not- $Y \mathrm{~s}$ " and "the proposition 'Some $X \mathrm{~s}$ are $Y \mathrm{~s}$ ' is false". He says that his preference of the former (complement) reading is "not a useless refinement but a necessary step, in order to make the proposition truly a relation between classes" (Boole's emphasis). Moreover, when he deals with what is now taken as negation of a proposition he again avoids negation by taking an elective symbol for a proposition to indicate not a truth-value but rather a class, viz. the class of "cases" in which the corresponding proposition is true (MAL, p. 49). Thus even in this context negation is supplanted by complementation. In Laws of Thought (pp. 164-165), Boole understands the elective symbol $x$ corresponding to the proposition $X$ to indicate "that portion of time for which the proposition $X$ is true", still avoiding negation.

7. Although Boole does not say so, the same two rules in exactly the same wording are found in Whately ([24], II, iii, 2).

8. In the above we have followed Boole's practice of using elective symbols in metalinguistic statements of axiom schemes and rules as if they were syntactic variables. The alternative, in regard to axioms, is to suppose that Boole implicitly assumed a general 
substitution rule (from $S$ infer any sentence obtained from $S$ by replacing all occurrences of a single elective symbol by one and the same term). To preserve consequence general substitution must be restricted so that it applies only to sentences derived from logical axioms (e.g., Corcoran, [9], p. 155). This virtually entails that the elective symbols are free variables, but there is no evidence in $M A L$ to support that conclusion. On the contrary, the elective symbols are repeatedly used to refer to "arbitrary but fixed" classes. In effect we are claiming that Boole's object language had no variables and thus is not an algebraic language in the usual sense (e.g., Craig, [13], Hailperin, [16]).

9. The additions suggested below are based on the supposition, made for illustrative purposes only, that + and - are interpreted as union and relative complement. Of course, if these two symbols are interpreted differently then other additions would be appropriate. For example, if + is taken as partial union then Boole's distributive law is not among the "propositions true in consequence of form alone" because if $x=\{1,2,3\}$, $u=\{1,4\}$ and $v=\{3,4\}$ the left side is undefined and the right side is $\{1,3\}$. The main point is that no matter how the two symbols are interpreted major additions are necessary. Incidentally, contrary to Kneale and Kneale ([18], p. 406), important additions are made in Laws of Thought. Also, in connection with U1 see MAL, p. 17n.

10. Wood ([26], pp. 195-196) has shown that if + and - are interpreted as the partial functions used in Laws of Thought then DeMorgan's Laws do not hold. If DeMorgan's laws are taken as conditions on two classes $x$ and $y$ then they imply that $x$ is the complement of $y$.

11. Boole was also not aware of the fact that Aristotle's rules do not admit of easy generalization into the richer framework. For example, Aristotle inferred "Some $A$ s are $B \mathrm{~s}$ " from "All $A \mathrm{~s}$ are $B \mathrm{~s}$ " because his terms were "logically simple" universals which are grasped through perception of individuals coming under them and which are, therefore, inherently nonempty. But, of course, terms which are compounded out of logically simple universals are not logically simple and thus may be empty. Thus for Aristotle, "Some $C A$ s are $B \mathrm{~s}$ " does not follow from "All $C A$ s are $B \mathrm{~s}$ ". For example, "Some circular squares are rectangles" does not follow from "All circular squares are rectangles" (Corcoran [11], pp. 103-104).

12. If one is solving equations $p(x, y)=q(x, y)$ one is asking either (1) whether there are objects $x$ and $y$ which satisfy the equation and if so what they are or (2) whether there are functions $x=f(y)$ which satisfy the equation (for all values of $y$ ) and if so what they are. In neither case can one assume that such objects or functions exist. If one is considering equations as expressive of propositions for purposes of drawing inferences then the letters $x$ and $y$ are taken as "names" of objects (or classes) and in this case it is absurd to speak of $x$ as a function of $y$. Could 7 be a function of 8 ? Could the class of men be a function of the class of animals? Nevertheless Boole confuses these distinct roles of letters $(M A L, \mathrm{pp} .70,71)$. In this location he thinks of an equation involving $x$ and $y$ as if it gives " $y$ as a function of $x$ " (MAL, pp. 24, 70 and cf. Langer [19], p. 359). This confusion has unfortunate consequences for Boole's "analytic method" which, however, is not to be discussed in this article.

In the passage quoted from $M A L$, pp. 26-28, Boole derives "Some $Y \mathrm{~s}$ are $X \mathrm{~s}$ " $[x=v y]$ from "All $X \mathrm{~s}$ are $Y \mathrm{~s}$ " $[x(1-y)=0]$ taking the latter as a condition on $x$ as a function of $y$. But if he had taken it as a condition on $y$ as a function of $x$ he would have seen that $y=x$ is a solution and thus that the equivalence of the $X$ s to the $Y$ s could be derived by his reasoning from "All $X$ s are $Y \mathrm{~s}$ ". 
13. A similar difficulty obtains if Boole is taking "-" as partial complement. In this case one must use both premises but the conclusion still depends only on the fact that $c+d=0$ implies $d=0$. Thus the strengthened scheme would be SPE: from $a y+b=0$ and $a^{\prime} y+b^{\prime}=0$ infer $z b^{\prime}-w b=0$. Here the conclusion is again independent of the coefficients of $y$.

14. If one is to apply E15, as stated by Boole, to $(1-x) y=0$ and $z y-z=0$ then one must construe the two as $(1-x) y-0=0$ and $z y+-z=0$ (and one must construe the result as $z(1-x)-z 0=0)$. Now, $z y+-z=0$ implies that $z y=0$ and that $-z=0$, neither of which follows from the original premises.

15. Boole read "--" as "except". He did not take up the question of whether "All $A$ s except $B$ s are $C$ s" implies "Some $A$ s except $B$ s are $C$ s".

16. As indicated above, Boole had one logical system which he tried to use to treat both syllogistic and truth-functional reasoning. But, it does not seem to have occurred to him that there are arguments which involve a combination of the two, e.g., "All $A$ s are Bs" implies "Either All $B$ s are $C$ s or Some $A$ s are not $-C \mathrm{~s}$ ". It is well-known that such arguments cannot be treated in Boole's system.

17. Boole's claim ([6], pp. 20, 211, 218) that the "laws" of his algebra are exactly the sentences which are true on arbitrary substitutions of 0 and 1 seems to qualify him for mention in any history of decision procedures and in any other history of logic which emphasizes attempts to reduce reasoning to computing. Boole's discussions of the role of logical axioms in deduction ( $M A L$, pp. 5, 6, 18; [6], pp. 144, 215) qualify him for mention in any history of formal deduction.

18. The reader should notice that most of our criticisms of Boole's inattention to logical detail are independent of which interpretation Boole had in mind for + and - in $M A L$. Moreover, each particular charge which was supported by argument based on one interpretation applied to passages which contain mistakes relative to the other interpretation as well.

\section{REFERENCES}

[1] Anselm, De Grammatico [XIth c.], Latin text and English translation in D. Henry, Commentary on De Grammatico, Reidel Publishing Co., Dordrecht, Holland, 1974.

[2] Bacon, J., "Natural-deduction rules for syllogistic" (abstract), The Journal of Symbolic Logic, vol. 31 (1966), pp. 686-687.

[3] Boole, G., The Mathematical Analysis of Logic, Being an Essay Towards a Calculus of Deductive Reasoning, Macmillan, Barclay \& Macmillan, Cambridge and George Bell, London, 1847, also repinted 1948 by Basil Blackwell, London.

[4] Boole, G., An Investigation of the Laws of Thought on Which are Founded the Mathematical Theories of Logic and Probabilities, Macmillan, London, 1854. Pagination referred to here is from the 1954 Dover reprint.

[5] Boole, G., A Treatise on Differential Equations, Cambridge, 1859. Pagination referred to from reprint of the fifth edition by Chelsea Publishing Co., New York.

[6] Boole, G., Studies in Logic and Probability, edited by R. Rhees, Open Court Publishing Company, LaSalle, Illinois and Watts \& Co., London, 1952.

[7] Church, A., Introduction to Mathematical Logic, Princeton University Press, Princeton, N.J., 1956. 
[8] Cohen, P. and R. Hersh, "Non-Cantorian set theory," Scientific American, vol. 217 (1967), pp. 104-116.

[9] Corcoran, J., "Three logical theories," Philosophy of Science, vol. 36 (1969), pp. 153-177.

[10] Corcoran, J., "Completeness of an ancient logic," The Journal of Symbolic Logic, vol. 37 (1972), pp. 696-702.

[11] Corcoran, J., "Aristotle's Natural Deduction System" in Ancient Logic and Its Modern Interpretations, ed., J. Corcoran, Reidel Publishing Co., Dordrecht, Holland, 1974.

[12] Corcoran, J. and S. Wood, "Validity and invalidity: Boole's criteria" (abstract), The Journal of Symbolic Logic, vol. 44 (1979), p. 662.

[13] Craig, W., Logic in Algebraic Form, North-Holland, Amsterdam, 1974.

[14] DeMorgan, A., Formal Logic, ed., A. E. Taylor, Open Court Co., London, 1926, orig. publ. Taylor and Walton, London, 1847.

[15] Dummett, M., Review of [6], The Journal of Symbolic Logic, vol. 24 (1959), pp. 203-209.

[16] Hailperin, T., Boole's Logic and Probability, North-Holland, Amsterdam, 1976.

[17] Henry, D., Commentary on De Grammatico, Reidel Publishing Co., Dordrecht, Holland, 1974.

[18] Kneale, W. and M. Kneale, The Development of Logic, Oxford University Press, Oxford, 1962.

[19] Langer, R. E., "Differential equations," Encyclopedia Brittanica, 1953 edition, vol. 7, pp. 358-363.

[20] Mill, John Stuart, A System of Logic, Longmans, Green and Company, London, 1879, originally published in 1843 .

[21] Smiley, T., "What is a syllogism?," Journal of Philosophical Logic, vol. 2 (1973), pp. 136-154.

[22] Tarski, A., Introduction to Logic and to the Methodology of Deductive Sciences, tr., O. Helmer, Oxford University Press, New York, 1941.

[23] Van Evra, J., "A reassessment of George Boole's theory of logic," Notre Dame Journal of Formal Logic, vol. 18 (1977), pp. 363-377.

[24] Whately, R., Elements of Logic, James Munroe \& Co., Boston, 1845, originally published in London, 1825.

[25] Whitehead, A. N., Universal Algebra, vol. 1, Cambridge University Press, Cambridge, 1898.

[26] Wood, S., George Boole's Theory of Propositional Forms, Ph.D. Dissertation in Philosophy, SUNY/Buffalo, 1976.

State University of New York at Buffalo

Buffalo, New York, 14260

and

State University of New York at Stony Brook

Stony Brook, Long Island, New York, 11794 